\title{
Linear and Nonlinear Regime of a Random Resistor Network Under Biased Percolation
}

\author{
C. Pennetta, E. Alfinito and L. Reggiani \\ INFM National Nanotechnology Laboratory and Dipartimento di Ingegneria \\ dell'Innovazione, Università di Lecce, Via Arnesano, 73100 Lecce, Italy
}

\begin{abstract}
We investigate the steady state of a two-dimensional random resistor network subjected to two competing biased percolations as a function of the bias strength. The properties of the linear and nonlinear regimes are studied by means of Monte Carlo simulations. In constant current conditions, a scaling relation is found between $\left\langle R>/\left\langle R>_{0}\right.\right.$ and $I / I_{0}$, where $\langle R\rangle$ is the average network resistance, $\left\langle R>_{0}\right.$ the Ohmic resistance and $I_{0}$ an appropriate threshold value for the onset of nonlinearity. A similar scaling relation is found also for the relative variance of resistance fluctuations. These results are in good agreement with electrical breakdown measurements performed in composite materials.
\end{abstract}

The study of electrical and mechanical stability of disordered systems is attracting a considerable interest because of its implications on both the material technology [1, 2] and fundamental aspects related to the understanding of the behavior of these systems 11, 3, 4, 5]. Indeed, the application of a finite stress (electrical or mechanical) to a disordered material generally implies a nonlinear response, which ultimately leads to an irreversible breakdown in the high stress limit [1, 3]. Such catastrophic phenomena have been successfully studied by using percolation theories [1, 3, 4, 5, 6. In particular, large attention has been devoted to the critical exponents describing the resistance and its relative noise in terms of the property of the medium [1, , 6, 7]. However, few attempts have been made so far to describe the behavior of a disordered media over the full range of the applied stress [8, 9]. On the other hand, such a study can provide important information about precursor effects and, more generally, about the predictability of breakdown [8, 9]. Therefore, a satisfactory understanding of breakdown phenomena is demanding [1, 3].

Here, our aim is to present a percolative model of sufficient generality to address the above issues. Our goal is to provide a theoretical framework to study response and fluctuation phenomena under linear and nonlinear regimes in a wide class of disordered systems. To this purpose, we analyze the evolution of a random resistor network $(\mathrm{RRN})$ in which two competing percolative processes are present, defect generation and defect recovery. These processes, which determine the values of the elementary network resistances, are driven by the joint effect of an electrical bias and of the heat exchange with a thermal bath. The bias is set up by applying a constant current. Monte Carlo (MC) simulations are performed to investigate the network evolution in the full range of bias values. Indeed, depending on the bias strength, an irreversible failure or a steady state of the RRN can be achieved. By focusing on the steady state, we analyze the behavior of the average network resistance, $\langle R\rangle$, and the properties of the resistance fluctuations, as a function of the bias. 
The paper is organized as follows. In Sect. 1 we briefly describe the model used. Section 2 presents the results of the MC simulations for the resistance and its fluctuations. The main conclusions are drawn in Sect. 3

\section{The model}

We study a two-dimensional random resistor network of total resistance $R$, made of $N_{\text {tot }}$ resistors, each of resistance $r_{n}$, disposed on a square lattice. We take a square geometry, $N \times N$, where $N$ determines the linear size of the lattice. For the comparison with resistance measurements of thin films, the value of $N$ can be related to the ratio between the size of the sample and the average size of the grains composing the sample. An external bias represented by a constant current $I$, is applied to the RRN through electrical contacts realized by perfectly conducting bars at the left and right hand sides of the network. A current $i_{n}$ is then flowing through each resistor. The RRN interacts with a thermal bath at temperature $T_{0}$ and the resistances $r_{n}$ depend linearly on the local temperatures, $T_{n}$, as:

$$
r_{n}\left(T_{n}\right)=r_{0}\left[1+\alpha\left(T_{n}-T_{0}\right)\right]
$$

In this expression $\alpha$ is the temperature coefficient of the resistance, and $T_{n}$ is calculated by adopting a biased percolation model [10] as:

$$
T_{n}=T_{0}+A\left[r_{n} i_{n}^{2}+\frac{B}{N_{\text {neig }}} \sum_{l=1}^{N_{\text {neig }}}\left(r_{l} i_{l}^{2}-r_{n} i_{n}^{2}\right)\right]
$$

Here, $N_{\text {neig }}$ is the number of first neighbours around the $\mathrm{n} t h$ resistor, the parameter $A$ describes the heat coupling of each resistor with the thermal bath and it determines the importance of Joule heating effects. The parameter $B$ is taken to be equal to $3 / 4$ to provide a uniform heating for the case of perfect network. In the initial state (corresponding to the perfect network with no heating) all the resistors are identical: $r_{n} \equiv r_{0}$. Accordingly, the initial value of the resistance is $R_{0}=r_{0}[N /(N+1)]$. Now, we assume that two competing percolative processes act to determine the RRN evolution. The first process consists of generating fully insulating defects (resistors with very high resistance, i.e. broken resistors) with probability $W_{D, n}=\exp \left[-E_{D} / K_{B} T_{n}\right]$, where $E_{D}$ is an activation energy characteristic of the defect and $K_{B}$ the Boltzmann constant [10]. The second process consists of recovering the insulating defects with probability $W_{R, n}=\exp \left[-E_{R} / K_{B} T_{n}\right]$, where $E_{R}$ is an activation energy characteristic of this second process. For $A \neq 0$, Eq. (2) implies that both the processes (defect generation and defect recovery) are correlated percolations. Indeed, the probability of breaking (recovering) a resistor is higher in the so called "hot spots" of the RRN. On the other hand, for $A=0$ Eq. (22) yields $T_{n} \equiv T_{0}$, which corresponds to random percolations [6, 11]. The same is true for vanishing small bias values, when Joule heating effects are negligible. As a result of the competition between these two percolations the RRN reaches a steady state or fails completely with a critical fraction of defect $p_{c}$, corresponding to the percolation threshold [6]. In the first case, the network resistance fluctuates around an average value $\langle R\rangle$, while in the second case, an irreversible breakdown occurs, i.e. $R$ diverges due to the existence of at least one continuous path 


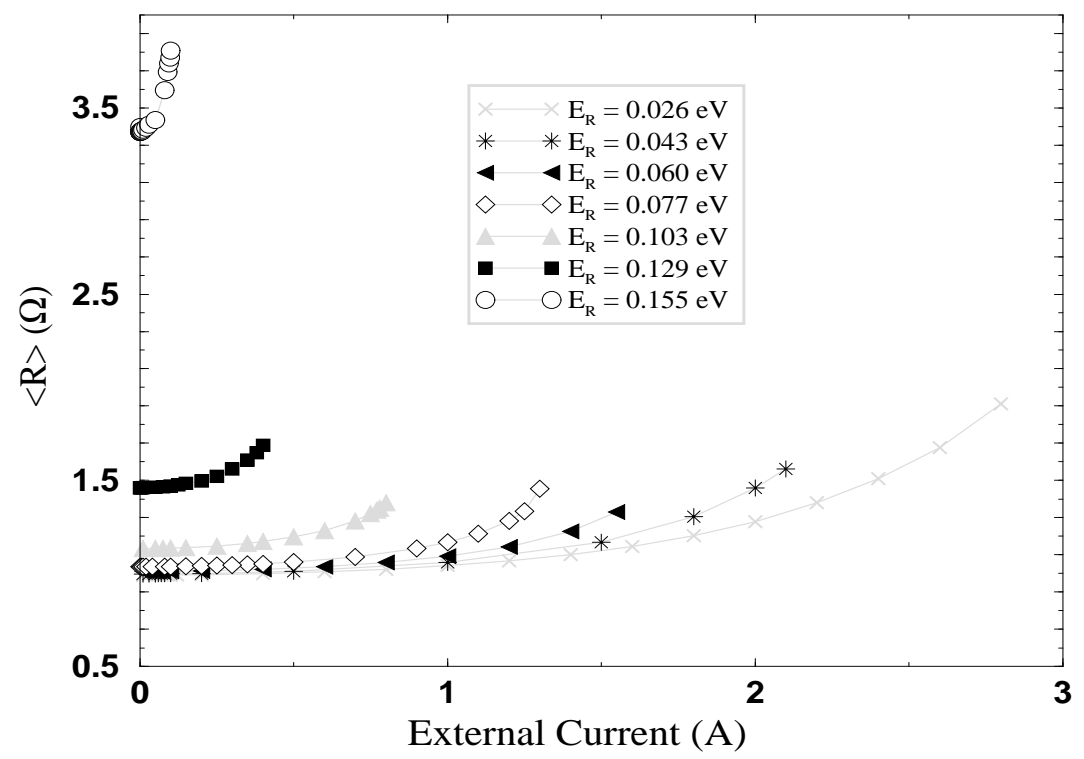

Figure 1: Average resistance versus the external current. Each set of data refers to the $E_{R}$ values shown in the legend.

of defects between the upper and lower sides of the network [6]. We note that in the limit of a vanishing bias (random percolation) and infinite lattices $(N \rightarrow \infty)$, the expression: $E_{R}<E_{D}+K_{B} T \ln \left[1+\exp \left(-E_{D} / K_{B} T\right)\right]$ provides a sufficient condition for the existence of a steady state [11], while it represents only a necessary condition in the case of biased percolation.

The evolution of the RRN is obtained by MC simulations carried out according to the following iterative procedure. (i) Starting from the perfect lattice with given local currents, the local temperatures $T_{n}$ are calculated according to Eq. (2); (ii) the defects are generated with probability $W_{D, n}$ and the resistances of the unbroken resistors are changed as specified by Eq. (11); (iii) the currents $i_{n}$ are calculated by solving Kirchhoff's loop equations by the Gauss elimination method and the local temperatures are updated; (iv) the defects are recovered with probability $W_{R, n}$ and the temperature dependence of unbroken resistors is again accounted for; $(\mathrm{v}) R, i_{n}$ and $T_{n}$ are finally calculated and the procedure is iterated from (ii) until one of the two following possibilities is achieved. In the first, electrical breakdown occurs (in numerical calculations we stop the iteration when $R$ increases over a factor of $10^{3}$ with respect to its initial value). In the second, the RRN reaches a steady state; in this case the iteration runs long enough to allow a fluctuation analysis to be carried out. Each iteration step can be associated with an elementary time step on an appropriate time scale (to be calibrated with experiments). In the simulations, as reasonable values of the parameters, we have taken: $N=75, r_{0}=1(\Omega), \alpha=10^{-3}\left(\mathrm{~K}^{-1}\right), A=5 \times 10^{5}$ $(\mathrm{K} / \mathrm{W})$ and $T_{0}=300(K)$ if not stated otherwise. Furthermore, $E_{D}=0.17(\mathrm{eV})$ and values of $E_{R}$ ranging from $0.026(\mathrm{eV})$ to $0.155(\mathrm{eV})$ are used. The values of the external bias range from $10^{-4} \leq I \leq 2.8(\mathrm{~A})$. 


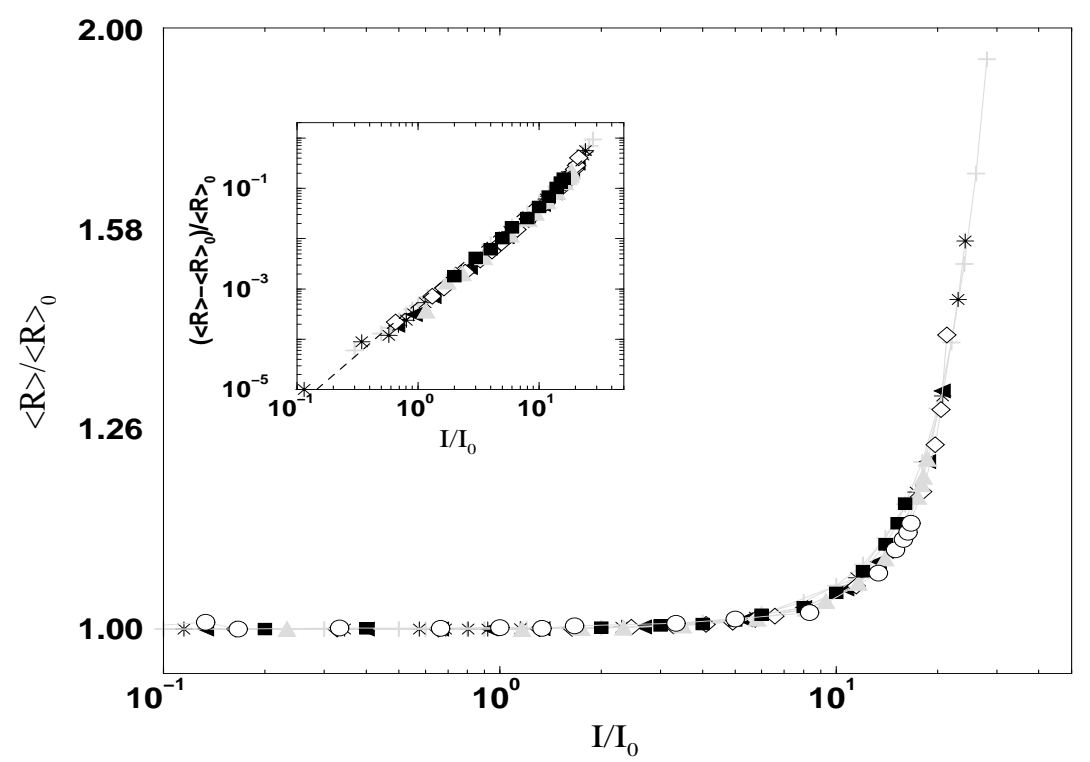

Figure 2: Log-log plot of the normalized resistances versus the normalized bias. Each set of data refers to different values of $E_{R}$ as reported in the legend of Fig. 1. The insert reports the quantity $\left(\langle R\rangle-\left\langle R_{0}\right\rangle\right) /\left\langle R_{0}\right\rangle$ and the dotted line fits the power law $y \propto x^{n}$ with $n=2$.

\section{Results}

Figure 1 reports the mean value of the RRN resistance as a function of the external current for the values of $E_{R}$ reported in the figure. Each value of $\langle R\rangle$ has been calculated by consider the time average on a single realization and then averaging over 20 realizations. The general behavior of each set of data shows a systematic increase of the resistance up to the breakdown of the network which occurs for currents greater than the breakdown value $I_{b}$ corresponding to the last point reported in the figure. For each set of data, at increasing current values, we identify three main transport regimes, namely: a linear regime at the lowest currents, a nonlinear regime at intermediate current values, and a pre-breakdown regime at the highest currents. The linear regime corresponds to a resistance which is independent of current (Ohmic response) and extends wider the lower the recovery energy. The nonlinear regime can be described by a quadratic dependence of the resistance in an appropriate range of current values starting from a threshold value $I_{0}$ and will be analyzed with details in the following. The pre-breakdown regime covers a narrow region just before the breakdown of the network which will be better analyzed on the basis of the resistance fluctuations. The fact that by increasing the recovery energy the breakdown occurs at lower currents is explained by the concomitant growth of the average number of defects, and thus of the disorder, inside the network which implies its lower stability against breakdown.

The similar feature of the data reported in Fig. 1 suggests the possibility that all the curves belong to the same universality class. We define the value of the current $I_{0}$ by adopting the criterium of the departure of the resistance from the linear response value of $0.05 \%$. Accordingly, Fig. 1 has been redrawn on a normalized current scale $I / I_{0}$ as is reported in Fig. 2. This figure, by showing that all curves collapse into a single one within an uncertainty of a few percent, clearly confirms the above suggestion. Furthermore, to obtain a better insight of the quadratic regime, in the insert we report 


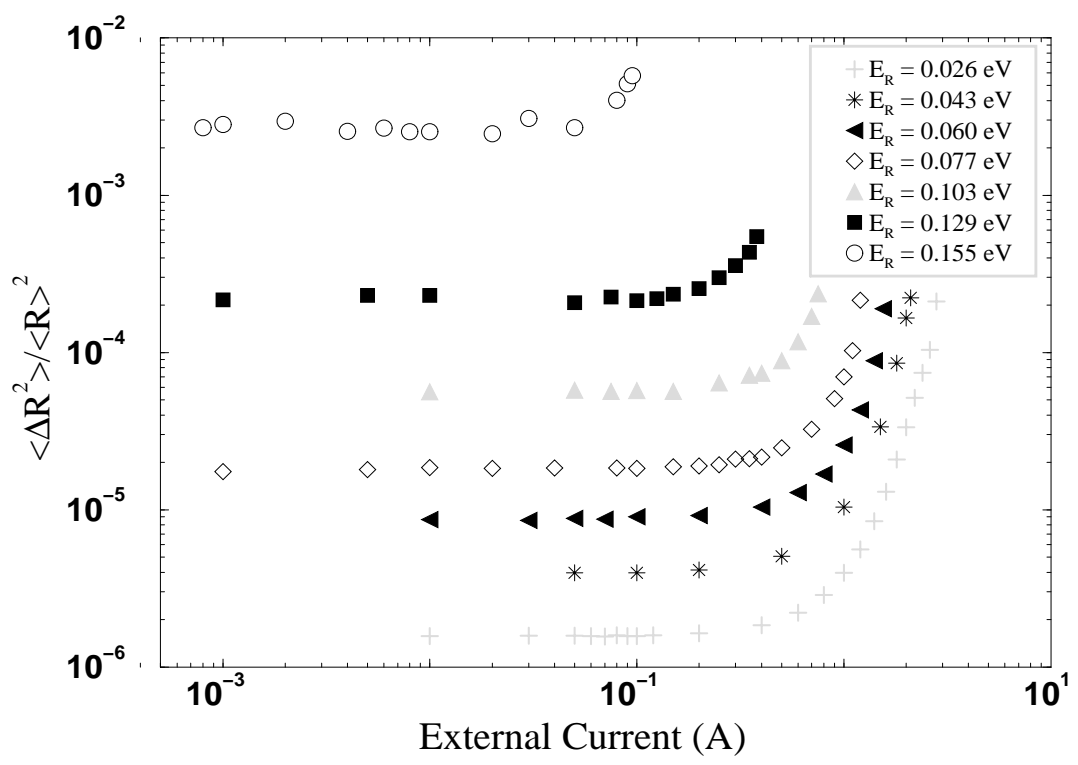

Figure 3: Log-log plot of the relative variance of resistance fluctuations versus the external current. Each set of data refers to the $E_{R}$ values shown in the legend.

the relative variation of resistance $\left(\langle R\rangle-\left\langle R_{0}\right\rangle\right) /\left\langle R_{0}\right\rangle$ as a function of the normalized current. Here, we have found that the power law:

$$
\frac{R(I)}{R_{0}}=g\left(\frac{I}{I_{0}}\right), \quad \text { with } \quad g\left(\frac{I}{I_{0}}\right)=1+\left(\frac{I}{I_{0}}\right)^{2},
$$

is well satisfied over about two decades of current values. The evidence of such a quadratic regime is in good agreement with recent experimental results [9] concerning the electrical failure of carbon high-density polyethylene composites (HDPE). In particular, their failure transition is found to be of the first order which is a feature we have checked in the present model. From the study of HDPE samples with different concentration of conductive component (carbon black) it was discovered that the ratio $R_{b} / R_{0}$ between the breakdown resistance $R_{b}$ and the Ohmic value $R_{0}$ is a constant independent of the value of the carbon fraction and the geometry of the sample, depending only on the nature of the conducting component. This result emerges, in our context, as a consequence of the independence of the ratio $I / I_{0}$ on the initial resistance [12] and of Eq.(33). The electrical properties of these composites are thus nicely described by a RRN modelling.

An important source of information concerning the physical properties of the stationary regime comes from the study of the resistance fluctuations. To this purpose we analyze in the following the variance of resistance fluctuations as obtained by the simulations. Figure 3 reports the relative variance of resistance fluctuations $\left\langle\Delta R^{2}\right\rangle /\langle R\rangle^{2} \equiv \Sigma$, with $\left\langle\Delta R^{2}\right\rangle=\left\langle R^{2}\right\rangle-\langle R\rangle^{2}$, as a function of the external current for the different values of recovery energy reported in the figure. The general behavior of different sets are similar to those reported in Fig. 1 for the resistance. Also in this case we find a systematic increase of the variance at increasing current with the remarkable novelty that now the increase can be of several order of magnitude for the most ordered network. By increasing the value of $E_{R}$, the curves start with higher values, in other words, the variance grows with $E_{R}$. Each curve is initially independent of current, 


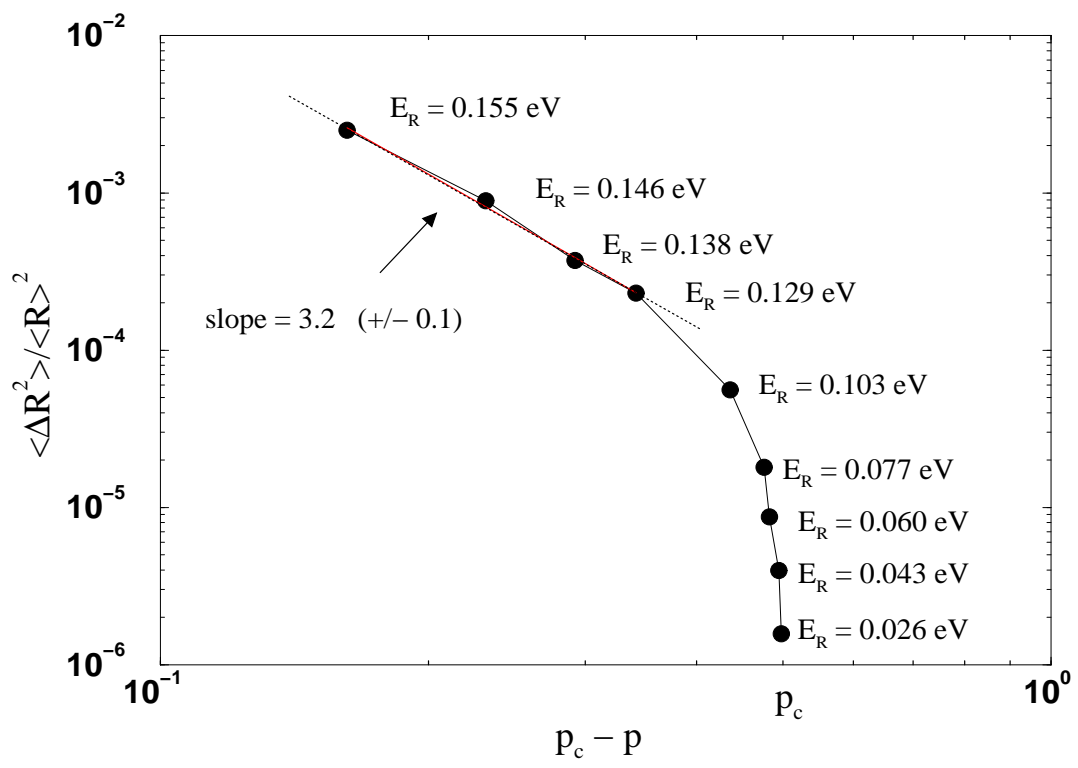

Figure 4: $\log -\log$ plot of the relative variance of resistance fluctuations versus $p_{c}-p$. All the values refer to the linear regime.

which corresponds to the linear regime where the variance is an intrinsic property of the RRN. Then, the variance starts increasing with current according with the establishment of the non-linear regime driven by the external current and accounted for by the bias percolation. Finally, the breakdown of the network is achieved but in a much sharp way than for the case of the resistance. We note, that the results at the two highest values of the recovery energy (open circles and full squares in the figure) are noisy already in the linear regime due to an initial network which is significantly disordered and thus less stable than those corresponding to low values of $E_{R}$.

The linear regime, occurring for small currents, when Joule heating effects are negligeable, practically corresponds to the competition of two random percolations [6]. We have thus investigated what kind of noise characterizes this random regime. Figure 4 reports the relative variance of resistance fluctuations $\Sigma$, as a function of the network defectiveness monitored by $p_{c}-p$ where $p_{c}=0.5$ is the critical value of the fraction of defects (i.e. the percolation threshold) for a square lattice and $p$ the average fraction of defects under stationary conditions. The plotted data have been calculated for bias $I<I_{0}$ and each point refers to the different value of $E_{R}$ reported in the figure. For recovery energies smaller than about $0.103(\mathrm{eV})$ we obtain a quasi-perfect lattice, i.e. $p \ll p_{c}$, while for larger values, the fraction of defects become significant and $\Sigma$ grows following the scaling law: $\Sigma \propto\left(p_{c}-p\right)^{k}$. Our simulations give $k=3.2 \pm 0.1$, thus confirming previous findings obtained by taking the thermal coupling parameter $A=0$ [11]. We note, that in literature [7] the value $1.12 \pm 0.02$ has been found for the case of a flicker noise spectrum in disordered network subjected to random percolation. Therefore, the present noise should be interpreted as coming from the intrinsic source of fluctuations and with the property of being much more sensitive to the presence of disorder.

As for the case of the resistance, the similar features of the data reported in Fig. 3 suggest the possibility that all the curves belong to the same universality class. To 


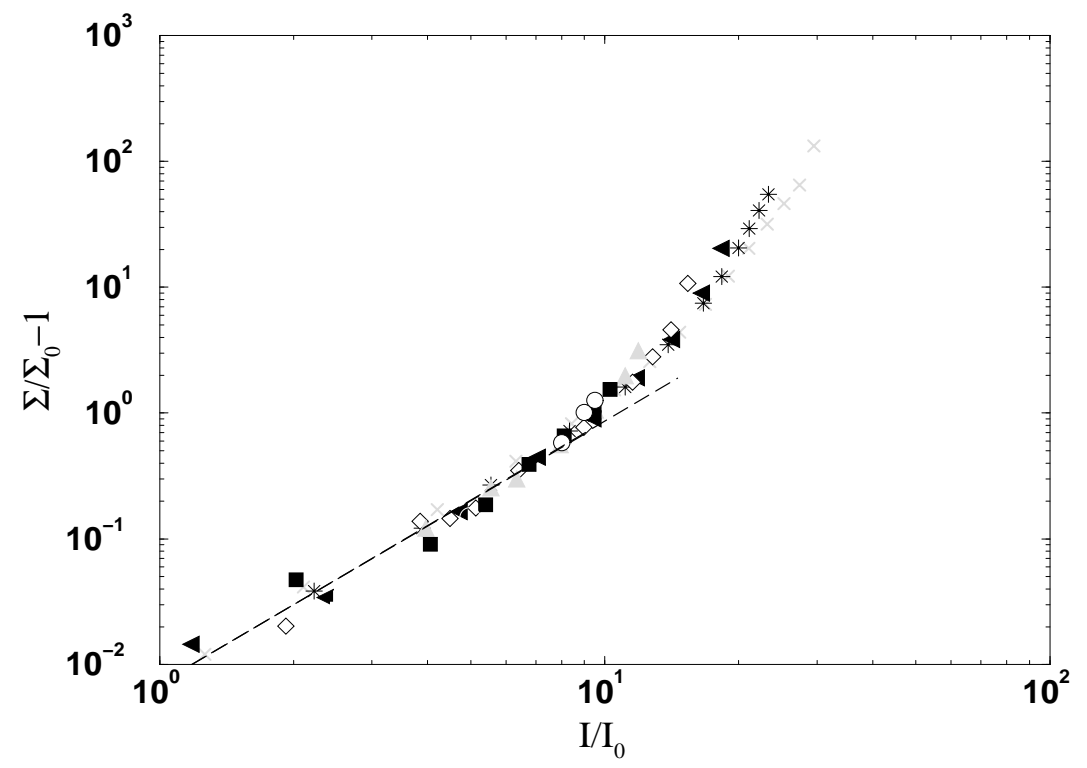

Figure 5: $\log -\log$ plot of $\Sigma / \Sigma_{0}-1$ where $\Sigma / \Sigma_{0}$ is the relative variance of resistance fluctuations normalized to the same quantity calculated in the linear regime. The dashed line emphasizes the region of validity of the quadratic power law.

this purpose, Fig. 5 reports on a $\log -\log$ scale the quantity $\Sigma / \Sigma_{0}-1$ as a function of the normalized current $I / I_{0}$. Here $\Sigma_{0}$ indicates the variance of resistance fluctuations for the Ohmic regime. The data are the same of those in Fig. 3 and all the curves are found to collapse onto a single one. Looking in detail the data in Fig. 5, we find that beside the quadratic regime, higher order terms are present [12]. Indeed, by analogy with the case of resistance, the quadratic regime is identified at the lowest current $\left(I<10 I_{0}\right)$ as emphasized by the continuous line. At high currents, $\left(I>10 I_{0}\right)$ a 4 -th order and eventually a 6 -th order regime can be identified. The presence of these high order terms can be accounted for by a unique law which expands the variance of resistance fluctuations in an even power series of the external current. In particular, for large values of $E_{R}$ the breakdown happens for small $I_{b}$, so only the quadratic term of the perturbative expansion, $\left(I / I_{0}\right)^{2}$, is appreciable. By contrast, for small values of $E_{R}$ the breakdown happens for large $I_{b}$ and terms of order higher than the second are detected. We conclude that the noise is more sensitive than resistance to probe the pre-breakdown region, since fluctuations are here dramatically amplified with respect to negligible deviations of the resistance from the quadratic regime.

\section{Conclusions}

We have studied the steady state of a two-dimensional random resistor network resulting from the simultaneous evolutions of two competing percolations biased from an external current. The two percolations consist in a defect generation process and in a defect recovery one. The state of the network is investigated by Monte Carlo simulations over the full range of the applied stress. In particular, we have analyzed the behavior of the average resistance and of the relative variance of resistance fluctuations as a function of the external current and for different values of the recovery activation 
energy. We have found that both these quantities follow a scaling relation in terms of $I / I_{0}$, i.e. the ratio between the applied current and the current value corresponding to the onset of nonlinearity. The scaling exponents are found to be independent of the activation energy value, implying that all the failure processes belong to the same universality class. In the linear regime, the relative variance of resistance fluctuations has been analysed with respect to the average fraction of defects in the network and, at increasing disorder, a scaling law as a function of $p_{c}-p$ has been obtained. In the nonlinear regime, the relative variance of resistance fluctuations exhibits a quadratic dependence on the current while the presence of higher order terms characterizes the pre-breakdown region. The analysis of these higher order terms is left as a subject of further investigation.

Acknowledgements

This research is performed within the STATE project of INFM. Partial support is also provided by the MADNESS II project of the Italian National Research Council and the ASI project N. 253.

\section{References}

[1] K. K. Bardhan, B. K. Chakrabarti, A. Hansen, Nonlinearity and breakdown in soft condensed matter, Springer-Verlag, New York (1994).

[2] M. Ohring Reliability and Failure of Electronic Materials and Devices, Academic Press, San Diego (1998).

[3] H. J. Herrmann, S. Roux, Statistical Models for the fracture of disordered media, North-Holland, Amsterdam (1990).

[4] A. Bunde, S. Havlin, Fractals and Disordered Systems, Springer-Verlag, Berlin (1996).

[5] H. E. Stanley, Rev. Mod. Phys., 71, 358 (1999).

[6] D. Stauffer, A. Aharony, Introduction to Percolation Theory, Taylor \& Francis, London (1992).

[7] R. Rammal, C. Tannous, P. Breton, A. M. S. Tremblay, Phys. Rev. Lett. 54, 1718 (1985).

[8] L. Lamaignère, F. Carmona, D. Sornette, Phys. Rev. Lett., 77, 2738 (1996).

[9] C.D. Mukherjee, K.K. Bardhan, and M.B. Heaney, Phys. Rev. Lett., 83, 1215 (1999).

[10] C. Pennetta, L. Reggiani, Gy. Trefan, Phys. Rev. Lett., 84, 5006 (2000).

[11] C. Pennetta, G. Trefan, L. Reggiani, Phys. Rev. Lett., 85, 5238 (2000).

[12] C. Pennetta, E. Alfinito, L. Reggiani, Journ. of Phys. C, in print. 